\title{
RESISTENCIA GENETICA Y CONTROL QUÍMICO DE LA ROYA DEL FRIJOL EN EL TRÓPICO HÚMEDO DE MÉXICO ${ }^{1}$
}

\author{
Enrique Becerra ${ }^{2}$, Ernesto López ${ }^{2}$, Jorge Acosta ${ }^{3}$
}

\begin{abstract}
RESUMEN
Resistencia genética y control químico de la roya del frijol en el trópico húmedo de México. La roya es una de las principales enfermedades del cultivo del frijol en el trópico húmedo de México. En mejoramiento gcnético, desde 1980 se han llevado a cabo evaluaciones de líneas avanzadas, pruebas preliminares y ensayos regionales de rendimiento y como resultado se liberaron en 1981 las variedades "Negro Veracruz", tolerante a roya, y "Negro Huasteco-81", resistente; y en 1991 se liberó la variedad "Negro Cotaxtla-91", resistente. En 1990 se iniciaron trabajos de evaluación de fungicidas para el control de la roya en varias localidades. El uso de fungicidas con variedades susceptibles como Jamapa, incrementó los rendimientos en más de $200 \mathrm{~kg} / \mathrm{ha}$. Los fungicidas que dieron los mejores resultados fueron: Hexaconazole $(0,5 \mathrm{l} / \mathrm{ha})$, Maneb (3 kg/ha) y Tehuconazole $(0,5 \mathrm{~kg} / \mathrm{ha})$. El costo de la aplicación de fungicidas se pagó con un incremento en el rendimiento de frijol de $75 \mathrm{~kg} / \mathrm{ha}$.
\end{abstract}

\begin{abstract}
Genetic resistance and cheroical control of bean (Phaseo/us vulgaris L.) rust in the Mexican tropics. The rust (Uromyces appendiculatus var. typica) is one of the main bean diseases in the humid Mexican tropics. The objective of this experiment is to present the results from the research conducted by the bean breeding program and the test of fungicides for controlling bean rust. Advanced lines' evaluation, preliminary trials and regional yield trials have been conducted since 1980 and as result, the rust tolerant "Negro Veracruz" and the rust resistant "Negro Huasteco-81" varieties were released in 1981. The rust resistant "Negro Cotaxtla-91" was released in 1991. Chemical rust control trials with fungicides were started at several localities in 1990. The application of fungicides on susceptible varieties like Jamapa inereased yields by over 200 $\mathrm{kg} / \mathrm{ha}$. The fungicides which best controlled bean rust were; Hexaconazole (0.5 1/ha), Maneb ( $3 \mathrm{~kg} / \mathrm{ha})$, and Tebuconazole $(0.5 \mathrm{~kg} / \mathrm{ha})$, when applied twice before blooming. The cost of the fungicide application was paid back by $75 \mathrm{~kg} / \mathrm{ha}$ of bean yield increase.
\end{abstract}

\section{INTRODUCCION}

El hongo Uromyces appendiculatus var. typica causante de la roya del frijol, es un patógeno que ha mostrado extensa variabilidad genética en las áreas productoras de frijol (Stavely et al., 1989) y constituye uno de los factores limitantes en el cultivo de frijol en diversas regiones de México. Estudios llevados a cabo para determinar las pérdidas en rendimiento causadas por este patógeno en el estado de Veracruz, han mostrado que pueden llegar al 30\% en la variedad "Mantequilla Tropical" (Yoshii, 1981) y a $25 \%$ en la variedad "Jamapa" (Becerra, 1992). En el Llano de Aguas Calientes, al comparar los rendimientos de frijol obtenidos en parcelas con y sin control químico de enfermedades, se obtuvieron rendimientos de 1, 100 y $250 \mathrm{~kg} / \mathrm{ha}$, respectivamente (Valle et al., 1987).

El uso de variedades resistentes o tolerantes a la roya ha sido la mejor opción para el control de la enfermedad en el trópico húmedo de México, pero debido a la variación patogénica del hongo y a la formación de nuevas razas, algunas variedades han ido perdiendo la resistencia.

1 Presentado en la XL Reunión Anual del PCCMCA en Costa Rica. América Cenlral. 13 al19 de marzo, 1994.

2 Investigadores del Programa de Leguminosas Comestibles. CIRGOC-INIFAP. C.E. "Cotaxtla". Apdo. Postal \#429. Veracruz. Ver. C.P. 91700. México.

3 Investigador del Programa de Leguminosas Comestibles. CIRCE-INIFAP. C.E. ;"Vallcde México". Apdo. Postal \#10. Chapingo, Edo. de México. C.P. 56230. México. 
En América Central se evaluó el control químico en contra del patógeno. siendo éste más efectivo durante el desarrollo vegetativo de la planta hasta antes de la noración (Yoshii y Gálvez, 1975). Los productos evaluados en contra de este hongo han sido preventivos como el Azufre (25 a $30 \mathrm{~kg} / \mathrm{ha}$ ), Parzate (2 kg/ha), Caldo Bordelés 0,5\%, Maneb (4-5 kg/ha), Mancozeb (3-4 kg/ha) y Nabam (2 kg/ha), y curativos como Benlate (300-400 g/ha), Plantvax $(2,5 \mathrm{~kg} / \mathrm{ha})$, Tiabendazol $(3,7 \mathrm{~kg} / \mathrm{ha})$, Baycor y Folicur (0,5 1/ha) (Zaumeyer 1946; Simbwa, 1973; Kantzes y Weaver, 1967, Zaumeyer y Gold-Sworthy, 1945, Crispin, et al., 1976; Vargas, 1980).

Otras prácticas que disminuyen la presencia de la enfermedad son: la rotación de cultivos, la eliminación de residuos de cosecha, la reducción de la densidad de siembra y el uso de fechas óptimas de siembra.

Dentro de las medidas de protección encaminadas a minimizar los daños causados por este patógeno en el trópico húmedo de México, se ha trabajado en la obtención de variedades resistentes y en la determinación de los mejores fungicidas y sus dosis para el control del hongo. Por lo anterior, el objetivo de este trabajo es dar a conocer los resultados de los estudios llevados a cabo para el control de la enfermedad durante 1980 a 1992.

\section{MATERIALES Y MÉTODOS}

Mejoramiento genético. Durante los años de 1980 a 1990 se trabajó en la evaluación de líneas avanzadas de frijol, tanto nacionales como introducidas, y en la selección masal e individual en poblaciones segregantes producidas por hibridación. Posteriormente, los mejores materiales fueron evaluados en pruebas preliminares y ensayos regionales de rendimiento, en diversas localidades de la región del trópico húmedo de México.

A partir de 1980 se inició la evaluación de un grupo de líneas avanzadas del programa local e introducidas de Guatemala y en el ciclo otoño-invierno (O-I) 1980-81 se llevó a cabo selección masal en las mejores. En el ciclo de invierno-primavera (I-P) 1981 se formó con las mejores líneas un ensayo regional y en el ciclo O-I 1981-82 un ensayo uniforme de rendimiento y adaptación. En 1983 se inició una serie de cruzamientos entre las mejores líneas y de la cruza D-149 X 1397 se originó la variedad "Negro Cotaxtla 91". El avance de la generación F1 se realizó en el ciclo primavera-verano (P-V) 1983, selección masal en la generación F2 en el ciclo I-P 1984, selección individual en F3 en el ciclo P-V 1984, compuesto masal F4 en O-I 1984-85 las pruebas preliminares de rendimiento en O-I 1986-87 y la evaluación en diferentes localidades del trópico húmedo de 1987 a 1990.

Evaluación de variedades y fungicidas.- Durante el ciclo P-V 1990, se evaluaron las variedades "Jamapa", "Negro Huasteco-81" y la línea experimental E-46, con y sin aplicación de fungicidas para el control de la roya en dos localidades, El Limonar y El Palmar, Veracruz. La protección química consistió en dos aplicaciones de cada uno de los fungicidas Hexaconazole $250 \mathrm{cc} / \mathrm{ha}$ y Maneb 2 $\mathrm{kg} / \mathrm{ha}$. Para evaluar los efectos de los tratamientos se cuantificó: reacción a roya a los 45 días después de la siembra (R6) y antes de la madurez (R8), altura de planta, granos por vaina, peso de 100 semillas, vainas normales por planta, vainas vanas, número de plantas cosechadas y rendimiento en $\mathrm{kg} / \mathrm{ha}$. La protección a roya se cuantificó mediante una escala de 5 grados, donde: $1=$ pocas o ninguna pústula (inmune); $2=20 \%$ de la superficie de las hojas con pústulas esporuladas (resistente); $3=40 \%$ de la superficie de las hojas con pústulas esporuladas (moderadamente resistentes); $4=60 \%$ de la superficie de las hojas con pústulas esporuladas (moderadamente susceptible) y $5=80 \%$ o más de la superficie de las hojas con pústulas esporuladas (susceptible).

En el I-P 1990-91 se continuó con el estudio en El Palmar y en Medellin de Bravo, Veracruz. En este experimento, además de los datos señalados en el párrafo anterior, en la localidad de Medellín de Bravo se determinó el Indice de Intensidad (II) de la roya en diferentes etapas fenológicas y su efecto sobre el rendimiento de plantas individuales. 
Durante el ciclo I-P 1991-92 se evaluaron en Cotaxtla, Veracruz, en la Variedad "Jamapa" cinco fungicidas: Hezaconazole 0,5 1/ha; Tebuconazole 0,5 1/ha; Maneb 3,0 $\mathrm{kg} / \mathrm{ha}$; Hidróxido cúprico $2,5 \mathrm{~kg} / \mathrm{ha}$ y Azufre líquido 2 1/ha. Se realizaron tres aplicaciones de cada uno de los productos a los 30, 39 y 49 días después de la siembra. La calificación de reacción a roya se hizo con la misma escala. Además, se cuantificó el rendimiento y el número de vainas por planta.

\section{RESULTADOS Y DISCUSION}

Mejoramiento genético.- Como resultado de los trabajos de mejoramiento, en 1981 se liberó la variedad "Negro Veracruz", tolerante a la roya. Su promedio de rendimiento fue de $1,517 \mathrm{~kg} / \mathrm{ha}$ en comparación con 1,300 de la variedad "Jamapa", susceptible a la roya (López y Vidal, 1981). La variedad "Jamapa" cuando se liberó en 1963 era tolerante a la roya, pero después de 20 años de uso se tornó susceptible. En 1982 se liberó la variedad "Negro Huasteco-81" con rendimiento promedio de 1440 $\mathrm{kg} / \mathrm{ha}$ en comparación con "Jamapa" de $950 \mathrm{~kg} / \mathrm{ha}$ y 970 de Negro Veracruz en 24 localidades durante 1980-81 (Yoshii y Rodríguez, 1982) la diferencia principal es la susceptibilidad al Virus del Mosaico Dorado del frijol (BGMV) de las variedades Jamapa y Negro Veracruz. La variedad "Negro Huasteco-81" era resistente a la roya, sin embargo, se ha observado en los últimos años que ha pasado a ser tolerante. En 1991 se liberó la variedad "Negro Cotaxtla-91", resistente a la roya (López y Rodríguez,
1992). Al comparar el rendimiento promedio obtenido en diferentes localidades y por varios años de las variedades liberadas "Negro Veracruz", "Negro Huasteco-81" y "Negro Cotaxtla-91 " con el de "Jamapa", se encontró que la diferencia de rendimiento es mayor en las variedades liberadas recientemente (Cuadro 1). Lo anterior puede deberse a una mayor resistencia a la roya de las variedades recien liberadas o a la pérdida de esta resistencia en "Negro Veracruz" y "Negro Huasteco 81".

\section{Evaluación de variedades y fungicidas.}

En el ciclo P-V 1990, sólo hubo diferencias altamente significativas $(\mathrm{P}<0,10)$ entre tratamientos para reacción a la roya. La variedad "Jamapa" sin protección química (SPQ), resultó con el valor más alto (4) mientras que con protección química $(\mathrm{CPQ})$ resultó más bajo $(2,33)$ junto con "Negro Huasteco-81" (2,33) y "Negro Cotaxtla-91" $(2,00)$, pero éstos últimos sin protección química (SPQ) (Cuadro 2) "Negro Huasteco-81" y "Negro Cotaxtla-91" resultaron con los valores más bajos $(1,83$ y 1,17 respectivamente) $\mathrm{CPQ}$. A pesar de que no hubo diferencia significativa entre tratamientos para el rendimiento, hubo diferencias numéricas con y sin la aplicación de fungicidas de 27 a $183 \mathrm{~kg} / \mathrm{ha}$ en la variedad "Negro Huasteco-81" y de 215 a 296 en "Jamapa" (Cuadro 3 y 4). La diferencia entre rendimiento de ambas localidades se debió principalmente a las condiciones de humedad, que fueron mejores en el Palmar, ya que en el Limonar se presentó sequía al

Cuadro 1. Porcentaje de rendimiento relativo de variedades obtenidas por el programa de mejoramiento genético, Cotaxtla, Ver. 1992.

\begin{tabular}{lcccc}
\hline Variedad & Reacción a Roya & \multicolumn{3}{c}{ \% de aumento en rendimiento en } \\
& & Jamparación con \\
& & $17 \%$ & N.V. & N.H.-81 \\
\hline Negro Veracruz & $\mathrm{T}$ & $49 \%$ & $45 \%$ &.-- \\
Negro Huasteco-81* & $\mathrm{T}$ & $15 \%$ & -- & $12 \%$ \\
Negro Cotaxtla-91 & $\mathrm{R}$ & & \\
\hline
\end{tabular}

$\mathrm{T}=$ Tolerante, $\mathrm{R}=$ Resistente.

* Problema principal de BGMV, además de roya. 
Cuadro 2. Reacción a roya y rendimiento de tres genotipos de frijol con y sin protección química en dos localidades de Veracruz. Ciclo P-V 1990.

\begin{tabular}{lcccccc}
\hline & & \multicolumn{2}{c}{ Reacción } & & \multicolumn{2}{c}{ Ren. kg/ha } \\
Genotipo & Protec. Quím. & El Palmar & El Limonar & & El Palmar & El Limonar \\
\hline JAMAPA & sin & $4,00 \mathrm{a} \&$ & $4,67 \mathrm{a}$ & & 1,673 & 769 \\
NH-81 & sin & $2,33 \mathrm{~b}$ & $2,00 \mathrm{c}$ & & 1,885 & 827 \\
JAMAPA & con & $2,33 \mathrm{~b}$ & $3,50 \mathrm{~b}$ & & 1,888 & 1,065 \\
NC-91 & sin & $2,00 \mathrm{~b}$ & $2,17 \mathrm{c}$ & & 2,036 & 852 \\
NH-81 & con & $1,83 \mathrm{bc}$ & $1,83 \mathrm{c}$ & & 1,912 & 1,010 \\
NC-91 & con & $1,17 \mathrm{c}$ & $1,50 \mathrm{c}$ & & 2,030 & 911 \\
Promedio & & 2,88 & 2,61 & & 1,904 & 906 \\
\hline
\end{tabular}

* = La proteccióm química consistió en dos aplicaciones de los fungicidas Hexaconazole ( $250 \mathrm{cc} / \mathrm{ha})$ y Maneb (2 kg/ha.)

$\&=$ Prueba de Duncan $\mathrm{P}<0,01$

Cuadro 3. Análisis marginal del rendimiento de tres genotipos de frijol con y sin protección química en contra de la roya en la localidad El Palmar. Ciclo P-V 90, Veracruz. México.

\begin{tabular}{|c|c|c|c|c|c|c|c|}
\hline \multirow[b]{2}{*}{ Genotipo } & \multirow[b]{2}{*}{ Quím.* } & \multirow[b]{2}{*}{$\begin{array}{l}\text { Rend. } \\
\text { kg/ha }\end{array}$} & \multicolumn{3}{|c|}{ Beneficio \& } & \multirow{2}{*}{$\begin{array}{l}\text { Relac. } \\
\text { Benef. } \\
\text { Costo }\end{array}$} & \multirow[b]{2}{*}{ TRM 1} \\
\hline & & & Costo & $\begin{array}{c}\text { Bruto } \\
\text {---Peso/ha²-- }\end{array}$ & Neto & & \\
\hline \multirow[t]{2}{*}{ JAMAPA } & $\sin$ & 1673 & 1152 & 3095 & 1944 & $2,69: 1$ & 184 \\
\hline & con & 1888 & 1292 & 3492 & 2202 & $2,70: 1$ & \\
\hline \multirow[t]{2}{*}{$\mathrm{NH}-81$} & $\sin$ & 1885 & 1152 & 3487 & 2336 & $3,03: 1$ & \\
\hline & con & 1912 & 1291 & 3537 & 2246 & $2,74: 1$ & \\
\hline \multirow[t]{2}{*}{ NC-91 } & $\sin$ & 2036 & 1152 & 3776 & 2615 & $3,27: 1$ & \\
\hline & con & 2030 & 1292 & 3775 & 2464 & $3,91: 1$ & \\
\hline
\end{tabular}

* = La protección química consistió en dos aplicaciones de los fungicidas Hexaconazole (250 cc/ha) y Maneb (2 kg/ha).

$\&=$ Precio del frijol $\mathrm{N} \$ 1,85 ; 1=\mathrm{TRM}=$ Tasa de retorno marginal; $2=3,11 \mathrm{~N} \$=1$ US \&

Cuadro 4. Análisis marginal del rendimiento de tres genotipos de frijol con y sin protección química en contra de la roya en la localidad El Limonar. Ciclo P-V 1990, Veracruz, Ver. México.

\begin{tabular}{|c|c|c|c|c|c|c|c|}
\hline \multirow[b]{2}{*}{ Genotipo } & \multirow[b]{2}{*}{ Química } & \multirow[b]{2}{*}{ Rend. kg/ha } & Costo & Bruto & Neto & \multirow{2}{*}{$\begin{array}{c}\text { Relación } \\
\text { Benef./Costo }\end{array}$} & \multirow[b]{2}{*}{ TRM 1} \\
\hline & & & & --Peso/ha ${ }^{2}--$ & & & \\
\hline \multirow[t]{2}{*}{ Jamapa } & $\sin$ & 769 & 1152 & 1422 & 272 & $1,24: 1$ & 291 \\
\hline & con & 1065 & 1292 & 1970 & 679 & $1,53: 1$ & \\
\hline \multirow[t]{2}{*}{$\mathrm{NH}-81$} & $\sin$ & 827 & 1152 & 1925 & 378 & $1,33: 1$ & 142 \\
\hline & con & 1010 & 1292 & 1868 & 577 & $1,45: 1$ & \\
\hline \multirow[t]{2}{*}{ NC-91 } & $\sin$ & 852 & 1152 & 1576 & 415 & $1,37: 1$ & \\
\hline & con & 811 & 1292 & 1683 & 394 & $1,30: 1$ & \\
\hline
\end{tabular}

* = La protección química consistió en dos aplicaciones de los fungicidas Hexaconazole (250 cc/ha) y Maneb (2kg/ha).

$\&=$ Precio del frijol N\$1,85; $1=\mathrm{TRM}=$ Tasa de retorno marginal; $2=\mathrm{N} \$ 3,11=1 \mathrm{US} \$$. 
momento de llenado de grano. En el análisis económico (Tasa de Retorno Marginal) se encontró que con incrementos de $75 \mathrm{~kg} / \mathrm{ha}$ se paga la aplicación de fungicidas. En el caso de la variedad "Negro Cotaxtla-91" no es necesario aplicar fungicidas ya que es resistente.

En el ciclo I-P 1991 se encontró en las dos localidades de prueba diferencias significativas $(\mathrm{P}>0,05)$ para la reacción a roya. En ambas, "Jamapa" obtuvo los valores más altos con y sin protección química y las demás variedades resultaron similares entre si bajo ambos tratamientos (Cuadro 5). En la localidad de El Palmar, la incidencia de roya fue ligeramente más alta que en Medellín de Bravo, en este sitio la incidencia del patógeno fue baja debido a condiciones ambientales prevalecientes durante el ciclo del cultivo de baja humedad relativa, menos de $80 \%$ y temperaturas menores de 15 o mayores de $30^{\circ}$ centígrados. Sin embargo, las variedades presentaron diferentes reacciones a la roya. El rendimiento sólo mostró diferencias significativas en la localidad de Medellín de Bravo. La variedad "Jamapa" bajo ambos tratamientos obtuvo los más bajos rendimientos, mientras que los más altos los obtuvo "Negro Cotaxtla-91" (Cuadro 5). Estos resultados concuerdan con lo obtenido por López y Rodríguez (1992), quienes encontraron que la variedad "Negro Cotaxtla-91" es más resistente y rinde más que Jamapa y "Negro Huasteco-81 ".

El Indice de Intensidad del ataque de la roya, resultó mayor durante la formación y llenado de vainas, sobre todo en las variedades "Jamapa" y "Mantequilla Tropical" (Cuadro 6). En estas variedades los porcentajes de roya resultaron altos desde las etapas fenológicas iniciales y se incrementaron a medida que se avanzó en el ciclo del cultivo.

Cuadro 5. Evaluación de tres variedades de frijol con y sin protección química y su reacción a roya, en el centro y norte del estado de Veracruz en el Ciclo I-P- 91-91. Veracruz, México.

\begin{tabular}{lcccccc}
\hline Variedades & Protección & \multicolumn{2}{c}{ Reacción a roya } & & \multicolumn{2}{c}{ Rendimiento kg/ha } \\
\cline { 3 - 4 } & química & Medellín & Palmar & & Medellín & Palmar \\
\hline Jamapa & sin & $2,4 \mathrm{a}^{* *}$ & $2,8 \mathrm{a}$ & & $465 \mathrm{a}$ & 1026 \\
Jamapa & con & $1,9 \mathrm{~b}$ & $2,1 \mathrm{~b}$ & & $430 \mathrm{~b}$ & 1072 \\
NH-81 & sin & $1,4 \mathrm{c}$ & $1,8 \mathrm{~b}$ & & $729 \mathrm{ab}$ & 1135 \\
NH-81 & con & $1,0 \mathrm{c}$ & $1,2 \mathrm{c}$ & & $640 \mathrm{ab}$ & 1076 \\
NC-91 & sin & $1,0 \mathrm{c}$ & $1,2 \mathrm{c}$ & & $932 \mathrm{a}$ & 1103 \\
NC-91 & con & $1,0 \mathrm{c}$ & $1,2 \mathrm{c}$ & & $886 \mathrm{a}$ & 1116 \\
\hline
\end{tabular}

* = La protección química consistió en dos aplicaciones de cada uno de los fungicidas Hexaconazole (250 cc/ha) y Maneb (2 kg/ ha).

$* *=$ Prueba de Duncan $\mathrm{P}<0,05$

Cuadro 6. Indice de Intensidad (I.I.) por estados fenológicos y variedades de frijol en el centro del estado de Veracruz. Ciclo I.P. 1991. Veracruz, México.

\begin{tabular}{lccccc}
\hline Variedad & Prefloración & Floración & Formación de vaina & Llenado de vaina & Promedio \\
\hline Jamapa & 43,00 & 38,42 & 50,00 & 59,47 & 47,72 \\
NH-81 & 28,33 & 31,25 & 40,47 & 28,57 & 32,15 \\
NC-91 & 20,00 & 20,00 & 33,52 & 18,57 & 23,02 \\
M. Tropical & 50,00 & 53,57 & 60,00 & 60,00 & 54,64 \\
\hline
\end{tabular}

I.I.= Número de observaciones de cada grado $\mathrm{X} \%$ de área con pústula/ número de observaciones. 
Los resultados obtenidos de la evaluación de cinco fungicidas en el ciclo I-P 1992-92 con la variedad "Jamapa", mostraron diferencias significativas para el rendimiento y número de vainas por planta (Cuadro 7). Los valores más altos para ambas características se obtuvieron con el fungicida Hexaconazole. Los productos que le siguieron en producción fueron Maneb y Tebuconazole, el más bajo rendimiento se obtuvo con el azufre líquido. Este y el Hidróxido cúprico resultaron similares al testigo. Para la calificación de roya se encontraron diferencias significativas $(\mathrm{P}<0,05)$ para la primera evaluación y altamente significativas $(\mathrm{P}<0,01)$ desde la segunda a la cuarta, destacando por su protección los fungicidas Hexaconazole y Tebuconazole (Cuadro 8). Estos resultados concuerdan con lo reportado por Ramírez (1991), quien encontró que los fungicidas sistémicos Bitertanol y Tebuconazole presentaron los menores porcentajes de áreas con pústulas de roya en Sonora, México.

\section{CONCLUSIONES}

Los trabajos de mejoramiento genético han producido variedades con buen nivel de resistencia y con adaptación a todas las áreas frijoleras del trópico húmedo de México, sin embargo, con el paso de los años las variedades han sucumbido ante la variación patogénica presentada por el hongo causante de la roya. Los resultados indican que la producción continua de materiales resistentes

Cuadro 7. Prueba de Duncan para rendimiento y número de vainas por planta de frijol en el centro de Veracruz. Ciclo I-P 1992-92. Veracruz, México.

\begin{tabular}{|c|c|c|}
\hline Tratamientos & Rendimiento (kg/ha) & No. vainas/plante \\
\hline Hexaconazole & $1142 \mathrm{~A}$ & $9,7 \mathrm{~A}$ \\
\hline Maneb & $958 \mathrm{AB}$ & $9,6 \mathrm{AB}$ \\
\hline Tebuconazole & $914 \mathrm{AB}$ & $8,4 \mathrm{ABC}$ \\
\hline Hidro. cúprico & $830 \quad \mathrm{~B}$ & $7,2 \mathrm{C}$ \\
\hline Testigo & $791 \quad \mathrm{~B}$ & $7,4 \mathrm{C}$ \\
\hline Azufre líquido & $727 \quad$ B & $8,0 \mathrm{BC}$ \\
\hline SIGNIFICANCIA & $*$ & * \\
\hline
\end{tabular}

* = Prueba de Duncan $\mathrm{P}<0,05$.

Cuadro 8. Prueba de Duncan para calificación de roya por fecha. Ciclo I.P. 1992-92. Veracruz, México.

\begin{tabular}{lcccc}
\hline Tratamientos & \multicolumn{4}{c}{ Calificaciones $\%$} \\
\cline { 2 - 5 } & $\mathbf{1}$ & $\mathbf{2}$ & $\mathbf{3}$ & $\mathbf{4}$ \\
\hline Hexaconazole & $13,0 \mathrm{~B}$ & $14,5 \mathrm{~B}$ & $16,5 \mathrm{CD}$ & $12,0 \mathrm{C}$ \\
Maneb & $14,2 \mathrm{~B}$ & $23,0 \mathrm{~A}$ & $30,3 \mathrm{AB}$ & $30,2 \mathrm{~B}$ \\
Tebuconazole 14,7 B & $15,7 \mathrm{~B}$ & $13,0 \mathrm{~B}$ & $11,0 \mathrm{C}$ & \\
Hidro. cúprico & $16,5 \mathrm{~B}$ & $27,7 \mathrm{~A}$ & $24,5 \mathrm{BC}$ & $29,5 \mathrm{~B}$ \\
Testigo & $15,0 \mathrm{~A}$ & $30,2 \mathrm{~A}$ & $33,7 \mathrm{~A}$ & $36,2 \mathrm{~A}$ \\
Azufre líquido & $20,0 \mathrm{~A}$ & $25,0 \mathrm{~A}$ & $34,5 \mathrm{~A}$ & $31,7 \mathrm{~A}$ \\
SIGNIFICANCIA & $* /$ & $* * /$ & $* * /$ & $* * /$ \\
\hline
\end{tabular}

*/ Prueba de Duncan $\mathrm{P}<0,05$.

**/ Prueba de duncan $\mathrm{P}<0,01$. 
a través del mejoramiento genético, es una de las mejores alternativas para el control de la enfermedad; sin embargo, además del simple cruzamiento entre una variedad susceptible y una resistente, se deben utilizar otras estrategias de mejoramiento como son la acumulación de diferentes genes de resistencia (Stavely et al., 1989), la introducción de genes de resistencia de diferentes razas o acervos genéticos a las variedades locales las cuales pertenecen a la raza mesoamericana (Singh, 1991), y la búsqueda de resistencia horizontal. Todo lo anterior para reducir el riesgo de un colapso de las nuevas variedades.

Por otro lado, en regiones favorables para el cultivo del frijol, en donde se espera un rendimiento relativamente alto el uso de fungicidas puede ayudar a garantizar una buena cosecha, principalmente, considerando que el patógeno causante de la roya puede presentar en un corto período de tiempo, nuevas variantes o razas en respuesta al uso extensivo de una variedad resistente. La combinación de una variedad tolerante con protección parcial con agroquímicos es también una posibilidad rentable para los productores de frijol del trópico húmedo de México.

\section{LITERATURA CITADA}

BECERRA, L. E.; LOPEZ, S. E.; RODRIGUEZ, R J. 1992. Cuantificación del daño ocasionado por la roya en el frijol en el estado de Veracruz. Resultados de Investigación sobre frijol 1991. Informe Técnico CIRGOC. Veracruz, Mex. p. 17-24.

CRISPIN, M. A.; SIFUENTES, A. J.; CAMPOS, A. J. 1976. Enfermedades y plagas del frijol en México. Folleto de DivulgaciónNo.39, INIA-SAG. p.42.

KANZES, J. G.; WEAVER, L. O. 1967. Chemical control of bean rusl. Phytopathology 57:646

LOPEZ, S. E.; RODRIGUEZ, R J. 1992. Negro Cotaxtla-91, nueva variedad de frijol para las zonas tropicales de Veracruz. Folleto técnico No. 1. CECOT. Veracruz, Mex. p. 8.

LOPEZ, S. E.; VIDAL, E. A. 1981. "Negro Veracruz" nueva variedad de frijol para las zonas central y sur de Veracruz (Descripción varietal). Folleto Técnico No.3 CAECOT. Veracruz, Mex. p.8.
RAMIREZ, A. J. 1991. Control químico de Uromyces phaseolitypica y Erysiphe polygoni en frijol en el Valle del Mayo, Sonora. XVIII Congreso Nacional de Fitopatología 24-26 de julio. Puebla, Méx.

SINGH, S. P.; GEPTS, P.; DEBOUCK, D. G. 1991. Races of common bean (Phaseolus vulgaris FABACEAE). Econ. Bol. 45:379-396.

SIMBWA-BUNNYA M. 1973. Part of preliminary report 00 fungicidal trials on beans at Kawanda. In: Uganda Kawanda Research Station. Annual Report 1972-1973. Uganda. p. 1-2.

STAVELY, J. R, STEADMAN, J. R; McMILLAN, RT. 1989. New pathogenic variability in Uromyces appendiculatus in North America. Plant Disease 73:428-432.

VALLE, P.; OCHOA, M. R.; RAMOS, R. F.; VILLALOBOS, J. J. 1987. Evaluación cuantitativa de pérdidas por enfermedades en frijol de temporal en Aguascalientes. Memoria del XIV Congreso Nacional de Fitopatología. 15-17 de julio, Morelia, Michoacán, Mex.

VARGAS, G. E. 1980. Rust. In: Bean production problems: disease, insect, soil and climatic constraints of Phaseolus vulgaris. Schwartz H.F. and Galvez G.E. (Eds). Centro de Investigación en Agronomía Tropical (CIAT). Cali, Colombia. p.17-36.

YOSHII, O. K. 1981. Enfermedades del cultivo de frijol en el sureste de México. IX Simposium Nacional de Parasitología Agrícola (IAP). 15-17 de Octubre, Mazatlan, Sin, Mex. p 52-74.

YOSHII, O. K.; GALVEZ, G. E. 1975. Effect of rusl on yield components of dry beans (Phaseolus vulgaris). Proc. Amer. Phytopathol. Soc.2:80 (Abstr.).

YOSHII, O. K.; RODRIGUEZ, R J. R. 1982. "Negro Huasteco81" nueva variedad de frijol para el trópico de México (Descripción varietal). Folleto No.1 CAECOT.Veracruz, Mex. p. 10.

ZAUMEYER, W. J. 1946. Field control of bean rust with sulfur. Phylopathology 36:689.

ZAUMEYER, W. J.; GOLDSWORTHY, M. C.1945. Control of bean rust by fungicide dusting and spraying.Phytopatholoy 35:489. 